\title{
Low Friction, Lubricity, and Durability of Polymer Brush Coatings, Characterized Using the Relaxation Tribometer Technique ${ }^{\dagger}$
}

\author{
Michel Belin ${ }^{1, *}$, Hiroyuki Arafune ${ }^{2}$ (D) , Toshio Kamijo ${ }^{2}$, Joël Perret-Liaudet ${ }^{1}$, \\ Takashi Morinaga $^{2}$, Saika Honma ${ }^{2}$ and Takaya Sato ${ }^{2}$ (iD \\ 1 Laboratoire de Tribologie et Dynamique des Systèmes, UMR 5513, École Centrale de Lyon, \\ Université de Lyon, 69134 Écully CEDEX, France; joel.perret-liaudet@ec-lyon.fr \\ 2 National Institute of Technology, Tsuruoka College, Tsuruoka 997-8511, Japan; \\ harafune@tsuruoka-nct.ac.jp (H.A.); kamijo@tsuruoka-nct.ac.jp (T.K.); morinaga@tsuruoka-nct.ac.jp (T.M.); \\ saika@tsuruoka-nct.ac.jp (S.H.); takayasa@tsuruoka-nct.ac.jp (T.S.) \\ * Correspondence: michel.belin@ec-lyon.fr; Tel.: +33-472-186-024 \\ $+\quad$ This paper is an extended version of our paper published in WTC 2017.
}

Received: 16 March 2018; Accepted: 1 May 2018; Published: 20 May 2018

\begin{abstract}
Among the possible solutions for achieving low friction, polymer brushes that are grafted onto surfaces are good candidates. The tribological characterization of such layers becomes more difficult when the friction is lower: the signal-to-noise ratio of the friction force that is measured with conventional tribometers impedes the precise quantification. Therefore, we have applied a new technique that has been developed at the Laboratory of Tribology and System Dynamics (LTDS), called the 'oscillating relaxation tribometer'. The advantage of this original technique is that it characterizes low friction with unequalled sensitivity. The lower the friction, the better the precision, and it permits obtaining the 'friction law' directly from robust and rapid experimental tests. In this study, the samples that have been used are the ionic liquid-type polymer brushes (ILPBs) with different thicknesses, which have been grafted onto silicon wafers and steel coupons. The counter-face is a mirror-polished steel ball. We show that (i) a thick ILPB layer on silicon is very resistant to high contact pressure, up to $555 \mathrm{MPa}$; (ii) the friction behavior that is obtained is close to that of a Newtonian viscous one, even under maximum normal loads; (iii) poorer results are obtained for the thinner sample; and (iv) the repetition, up to 5000 oscillations on the same surface, does not affect the friction damping of the contact, which demonstrates that this film provides a favorable resistance to friction under severe contact conditions. In addition, the feasibility of grafting onto steel surfaces is demonstrated. The results are then discussed, with respect to friction and dissipation.
\end{abstract}

Keywords: ultra-low friction; polymer brushes; ionic liquids; relaxation tribometer; damping

\section{Introduction}

Reducing friction to improve the performance and lifetime of the mechanical tribosystems that are used in fabrication and transportation is a challenging task. Much research has been devoted to developing robust low friction systems. Different approaches are possible, namely, the use of chemical additives to form a tribofilm on rubbing surfaces, the dispersion of nanoparticles in a fluid to prevent close contact between surfaces, and the use of anchored soft surface coatings [1,2]. Anchoring a polymer chain on the surface of materials is an efficient method for reducing the interfacial friction [3-6]. The polymer brushes are arrays of polymers whose ends are tethered to interfaces like substrates or particles. The development of the surface initiated radical polymerization method has made 
it possible to produce uniform and dense polymer brushes, using living radical polymerization [5]. This development has resulted in polymer brushes that theoretically fall into a 'concentrated' state when the concentration of the polymers in good solvents is high enough to eliminate the excluded volume effect, as with a concentrated solution that is of free polymers [6]. A concentrated state provides a highly extended chain conformation for polymer brushes whose equilibrium thickness reaches as much as $70-80 \%$ of the contour length of the grafted chains in good solvents. Such concentrated polymer brushes (CPBs) show unique properties, such as high compressive resistance and non-adhesiveness, compared with conventional ones (semi-diluted polymer brushes) [6]. They are also attractive as lubricants, since even the neutral brushes that are swelled in the organic solvents can exhibit 'superlubricity' with a friction coefficient $\mu$, typically $<5 \times 10^{-2}$ [7], without a hydration force or electrostatic repulsion, which are the main factors for inducing low friction in water $[2,8]$. However, most of the research utilizes water or organic solvents as swelling agents, however their volatility makes their application difficult for industrial usage.

Ionic liquids (ILs) are known to possess a high thermal stability, extremely low vapor pressure, non-flammability, and high ion conductivity, which are properties that have extended their application in various fields [9]. Since such properties are useful for lubricants [10], ILs are also suitable as swelling agents for polymer brushes, since they can maintain the swelling state of the latter under extreme conditions like long time exposure, high temperature, and vacuum. Several studies of such ionic liquid-type polymer brushes (ILPBs) have been reported [11,12]. Ishikawa et al. applied ILs for polymer backbones and lubricants in order to examine their lubrication properties [11]. A tribopair of a conventional glass ball and ILPBs, based on 1-ethyl-3-methylimidazolium bis(trifluoromethanesulfonyl)imide, resulted in efficient lubrication, which was maintained after 800 friction cycles, with a $\mu$ of around 0.13 . Sato and their coworkers developed a super lubrication system by using a molecularly smooth surface as a counterface [12]. In this system, they used a conventional glass ball and a smooth glass ball with maximum height differences $\left(S_{z}\right)$ of $220 \mathrm{~nm}$ and $2.4 \mathrm{~nm}$, respectively, as counter-faces for ILPB film (thickness: $40 \mathrm{~nm}$ ). While the relatively higher friction was observed in the case of the conventional glass balls, superlubrication was observed in the case of the smooth balls, which resulted in $\mu=0.003$ under $434 \mathrm{MPa}$. This result showed the importance of the surface of the counter-face in brush lubrication. In addition, this result suggests the possibility of superlubrication with the conventional tribomaterials, like steels, by applying a thicker polymer brush layer, exceeding the $S_{z}$ value of the counter-face, thereby limiting the abrasive wear.

In this study, we applied a tribopair of steels and thick polymer brushes, and characterized their lubrication properties to examine their friction regimes and durability. Although the literature includes many reports on brush lubrication with nanoscopic measurements, using surface force apparatus (SFA) and atomic force microscopy (AFM) [13-18], few reports have focused on macroscopic measurements. This may be because of the sensitivity of conventional macroscopic tribometers compared with the nanoscopic ones. We therefore use an oscillating relaxation tribometer to overcome this problem.

\section{Materials and Methods}

\subsection{Preparation of ILPBS}

ILPBs were formed on the $\mathrm{Si}$ and steel substrates, by the surface initiated-atom transfer radical polymerization (SI-ATRP), which was based on the literature [12]. A mixture of 3-((3-(triethoxysilyl)propyl)thio)propyl 2-bromo-2-methylpropanoate (BPTPE), ethanol, and aqueous ammonia was used to introduce the initiator moieties on the sample substrates. After rinsing the sample with ethanol, ATRP of the ionic liquid-type monomer, $N, N$-diethyl$\mathrm{N}$-(2-methacryloylethyl)- $\mathrm{N}$-methylammoniumbis(trifluoromethylsulfonyl)imide (DEMM-TFSI) was carried out with the BPTPE-modified sample substrate, using a degassed solution containing DEMM-TFSI, ethyl 2-bromoisobutyrate, $\mathrm{CuCl}, \mathrm{CuCl}_{2}$, and 2,2-bipyridine. The sample substrate was rinsed with acetonitrile so as to obtain the dried ILPB substrate, see schematic view in Figure 1. 
Two ILPB samples, with different dry thicknesses, were grafted onto a silicon wafer. The sample thicknesses on the silicon wafers $(620$ and $1860 \mathrm{~nm})$ with a surface density of $24 \%$, were characterized by the spectroscopic ellipsometry (M-2000, J.A. Woollam Co., Lincoln, NE, USA). An ionic liquid, DEMM-TFSI, was poured onto the dried ILPB substrate as a swelling agent and lubricant, before the friction measurement.

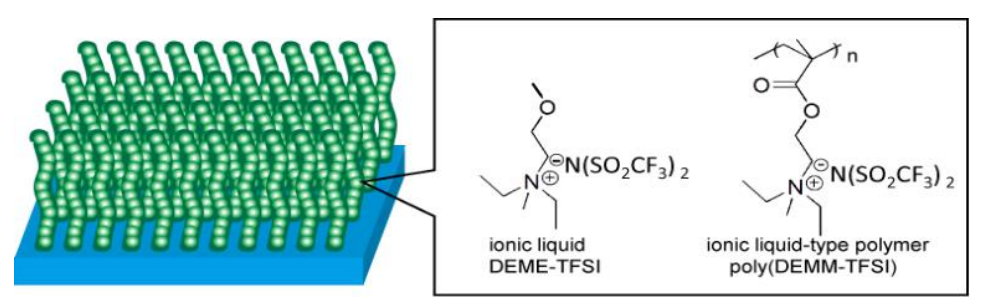

Figure 1. Schematic illustration of the ionic liquid-type polymer brushes (ILPBs).

\subsection{Testing with the Relaxation Tribometer}

The 'relaxation method' consisted of evaluating the friction without the direct measurement of the tangential force. The friction measurement was based on the analysis of the free-response velocity of a spherical pin in a sphere-on-plane contact. The spherical pin was connected to a support that was mounted on an elastic subsystem and the plane was fixed. The principle of the mechanical system is shown in Figure 2.

It consisted of a single degree-of-freedom (1-DOF) mechanical oscillator, which was damped by the rubbing contact. The double-leaf spring was made of steel bi-blades, and it allowed the spherical pin to oscillate horizontally, from an out-of-equilibrium initial position, that was imposed by an electromagnet. In other words, the electromagnet was used to initiate the movement and when it was deactivated, the spherical pin oscillated freely on the sample, until the stop was induced by the energy dissipation, as a result of the friction force. The normal load was applied by the deflection of an elastic spring, which supported the plane sample (not shown on Figure 2). It remained constant, while the spherical pin moved parallel to the plane until it stopped during the oscillatory test. The velocity time-response was measured using a laser velocimeter. In this operating mode, the mechanical system could be modeled by a simple 1-DOF mass-spring system, which allowed very rapid measurements typically lasting a few seconds. In addition, we could perform the measurement of the electrical contact resistance (ECR) between the two conducting solids during sliding. This real-time information could be useful for a better understanding of the actual contact and the lubrication regime.

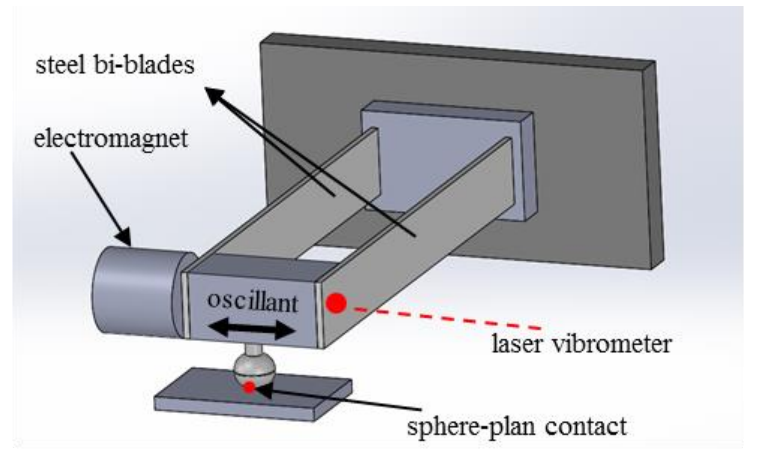

Figure 2. Schematic view of the oscillating relaxation tribometer.

\subsection{Identification of the Friction Laws}

In previous studies $[19,20]$, the authors had reported that the relaxation tribometer technique was able to discriminate between the different contributions to friction being, namely, velocity-dependent 
and velocity-independent. Another result showed that the evolution of friction depended on the velocity amplitude of the free oscillating response. On this basis, Majdoub et al. [21] proposed to determine the friction coefficient from the free-damped response of a sliding 1-DOF oscillator.

In the present study, we introduced a very simple way to achieve this, by assuming a constant friction coefficient at each oscillation. Thus, we considered the equation motion, written as follows:

$$
m \ddot{x}+k x= \pm \mu N
$$

where $x$ is the displacement, $\dot{x}$ is the sliding velocity, and $\ddot{x}$ is the corresponding acceleration. $m$ is the moving mass and $k$ the spring stiffness, $N$ is the applied normal load and $\mu$ is the kinetic friction coefficient that is to be determined. From this decaying amplitude of oscillations, we could obtain the friction coefficient in a classical way. The analysis of this response of a damped 1-DOF system has been described in detail by Hriouech et al. [22]. It has been shown that the decaying variation $\Delta \dot{x}_{3 p e a k s}$ between the three successive peaks of maximum velocity $\dot{x}$ was linked to the friction coefficient, by the following relation:

$$
\mu\left(V_{\text {max }}\right)=\Delta \dot{x}_{3 p e a k s} \frac{\sqrt{k m}}{8 N}, N \neq 0
$$

Before the experiments, we probed the intrinsic damping of the elastic system. It could have had different origins, namely, the internal damping of steel leaf material, air pumping, acoustic radiation, and mechanical dissipation in the assemblies. When no contact was applied, the elastic subsystem was released and gradually returned to equilibrium. The typical duration of this operation lasted more that $50 \mathrm{~s}$, which showed that the intrinsic damping of the oscillator was typically two orders of magnitude lower than that of the sliding loaded contact. Therefore, we considered in this study, that the intrinsic damping of the instrument could be neglected in data processing.

\section{Operating Conditions}

Silicon has been widely utilized as a substrate of polymer brushes, since the molecular surface smoothness was appropriate for the spectrometric characterization (ellipsometry) of polymer brushes, which led us to apply Si as a substrate. On the other hand, our objective in this paper was to characterize the applicability of the polymer brushes as a lubricant for industrial usage. We therefore applied steel as a counter-face substrate of the polymer brushes, since steel was one of the materials that was most commonly utilized for machines. Therefore, the experiment that was reported in this study consisted of a steel spherical pin (AISI 52100, from SNR-NTN, Annecy, France, with a diameter equal to $6.0 \mathrm{~mm}$ ), which slid on both the silicon wafer surface and a steel plane, with a mirror surface finish $(\mathrm{Ra}=30 \mathrm{~nm})$.

The contact is lubricated by a grafted ILPB layer swelled in a droplet, about $10 \mu \mathrm{L}$ of ionic liquid, $N, N$-diethyl- $N$-(2-methoxyethyl)- $N$-methylammoniumbis(trifluoromethylsulfonyl)imide (DEME-TFSI). This droplet of lubricant is naturally maintained in and around the contact due to capillary forces.

The experiments were performed in an ambient air in a temperature-controlled room at $25^{\circ} \mathrm{C}$. A normal load, ranging from 25 to $1000 \mathrm{mN}$, was applied, which led to an initial maximum contact pressure, which ranged from 160 to $680 \mathrm{MPa}$. In relaxation mode, the maximum velocity value $V_{\max }$ was $160 \mathrm{~mm} / \mathrm{s}$ and a duration of $8.0 \mathrm{~s}$ was chosen to record the free response. Each experiment in the relaxation mode was reproduced three times so as to check the repeatability of the oscillating time responses.

\section{Results and Discussion}

\subsection{Thick ILPB Grafted on Silicon}

We first performed tests on a 'thick' ILPB coating that was grafted onto a silicon surface. The normal load $N$ was gradually increased from 25 to $1000 \mathrm{mN}$. A set of three shots were performed in 
the same conditions, in order to check the variation in the velocity time-response of the loaded contact. Figure $3 a, c$ shows the typical evolution of the velocity with the time for 25 and $1000 \mathrm{mN}$ normal load, respectively, from the maximum velocity to the final stop. The general envelope of oscillations had an exponential appearance. As expected, the number of oscillations decreased when the normal force increased as a result of higher damping from the interface.

(a) $\mathrm{N}=25 \mathrm{mN}$

(b)
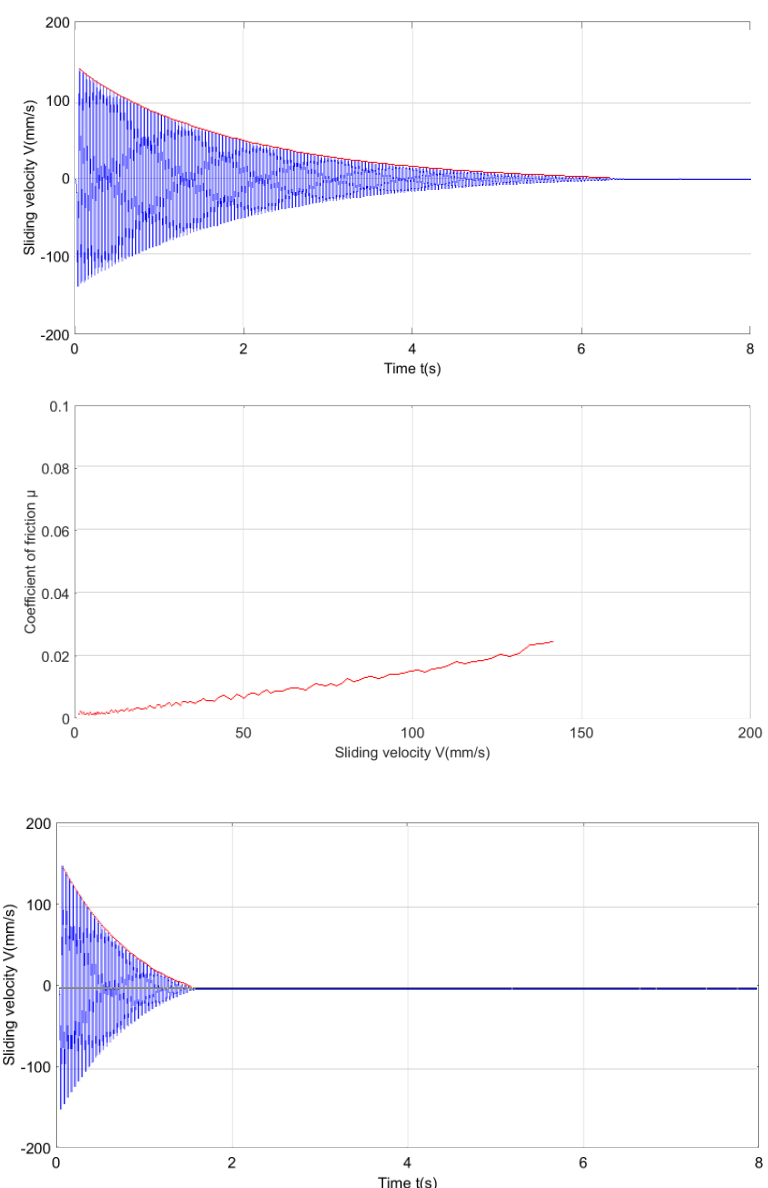

(c) $N=1000 \mathrm{mN}$

(d)

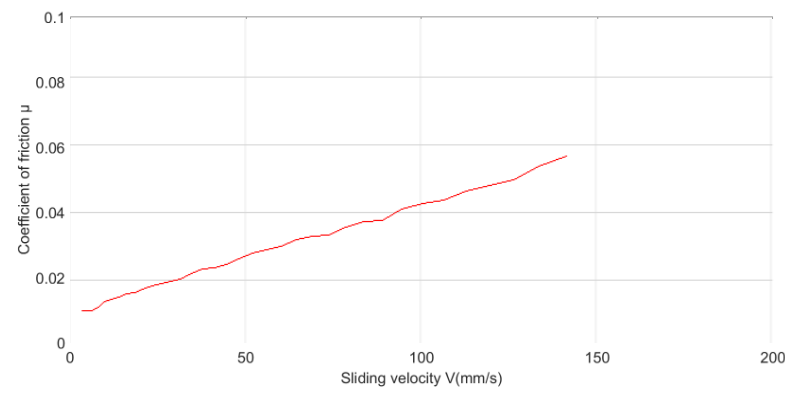

Figure 3. The typical evolution of the velocity of the oscillating sliding sphere with time $V=f(t)$ (in blue), and the envelope $V_{\max }=f(t)$ (in red), and the corresponding friction law diagram $\mu=f\left(V_{\max }\right)$-for two normal loads, $N=25 \mathrm{mN}(\mathbf{a}, \mathbf{b})$ and $1000 \mathrm{mN}(\mathbf{c}, \mathbf{d})$ for thick ILPB grafted onto silicon, tested with a steel sphere at $25^{\circ} \mathrm{C}$.

From this response, the envelope of maximum velocity was computed to extract the friction law. Figure $3 b$,d shows the evolution of the friction coefficient, which was calculated by the Equation (2) as a function of velocity. We observed that the friction coefficient decreased linearly with the maximum velocity. The corresponding evolution of the friction coefficient was plotted versus maximum sliding velocity $V_{\max }$. We found that $\mu$ had a linear dependence with the velocity, which corresponded to 
the viscous behavior of the interface. This confirmed that friction was an essentially viscous-type. In addition, we could deduce $\mu$ for $\mathrm{V} \rightarrow 0$, the value of the Coulomb type contribution, by extrapolating the friction law when the velocity reached a null value. In the case at $25 \mathrm{mN}$, it could be seen that $\mu$ for $\mathrm{V} \rightarrow 0$ was as low as 0.0008 , Figure 4 .

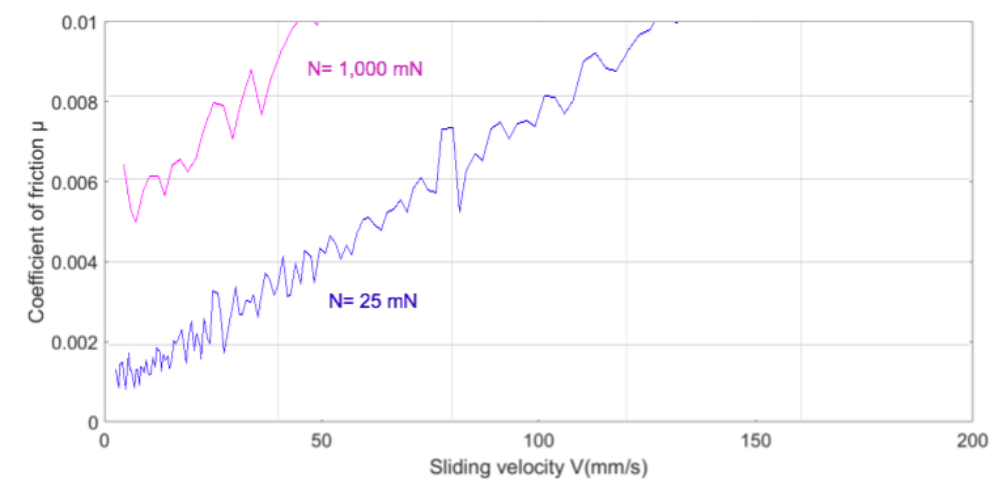

Figure 4. Detailed comparison of the friction laws $\mu=f\left(V_{\max }\right)$ that were computed in the two cases presented as follows: $N=25 \mathrm{mN}$ (blue) and $1000 \mathrm{mN}$ (pink). We showed that the Coulomb type friction was extremely low, less than 0.001 for the case of $25 \mathrm{mN}$.

\subsection{Thin ILPB Grafted on Steel}

The same type of experiment was performed on a 'thin' ILBP coating that was grafted onto a steel coupon. The normal load $N$ was gradually varied, from 25 to $500 \mathrm{mN}$. The typical velocity time-responses are presented in Figure 5, as follows: for (a) $25 \mathrm{mN}$, (b) $300 \mathrm{mN}$, and (c) $500 \mathrm{mN}$. In these cases, the shape of the envelope had changed, which showed that the friction behavior of the interface had changed.

We extracted the friction laws from these three typical curves, and the result is presented in Figure 6. Friction was found to be essentially viscous-type for the 25 and $300 \mathrm{mN}$ tests. Conversely, friction had increased considerably at $500 \mathrm{mN}$, which indicated a Coulomb type contribution. This curve also showed that the lubrication regime had changed from a full film regime to a classical boundary regime. This was probably because of a partial degradation of the coating, which led to the severe solid-solid contacting junctions in the contact zone.

In the case of a high normal load of $500 \mathrm{mN}$ (curve in red, in Figure 6), the general shape of a 'Stribeck curve' was found, which schematically represented the dependence of friction on the sliding velocity. The figure also showed the different lubrication regimes as a function of the value of the sliding velocity. It is noteworthy that the usual Stribeck curve $\mu=f(V)$ was defined and valid for a stationary velocity. In the relaxation tribometer technique, the friction law was determined in non-stationary conditions as a result of the dynamic effects of the varying velocity. Interestingly, the results that we had obtained here showed the same trend $\mu=f\left(V_{\max }\right)$. These tests were followed by decreasing the normal load oscillating shoots, which showed that this trend was not an artifact as a result of any topographic change of the sample surface or degradation of the coating. 
(a) $N=25 \mathrm{mN}$

(b) $N=300 \mathrm{mN}$
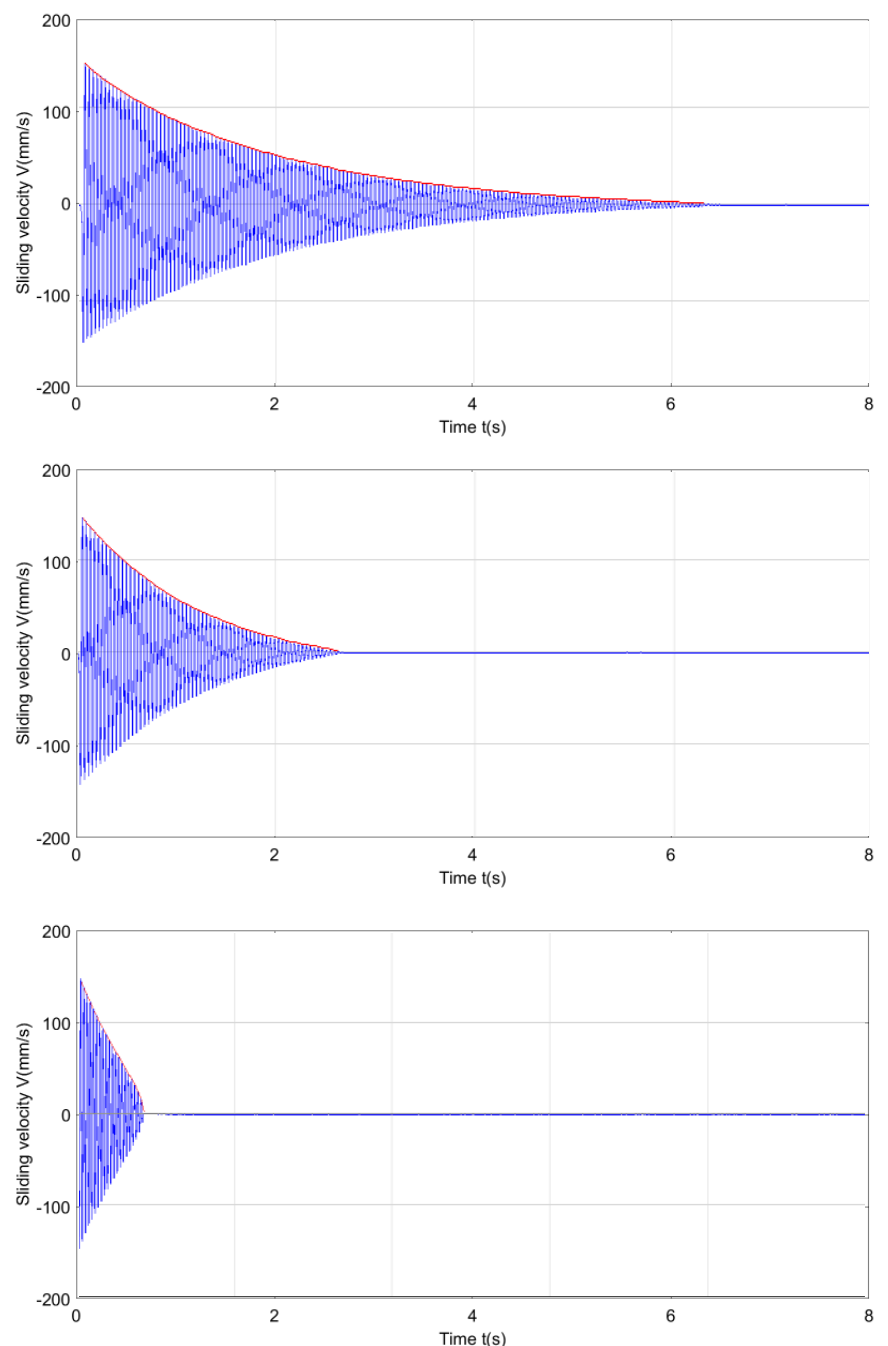

Figure 5. The typical evolution of the velocity of the oscillating sliding sphere, with time $V=f(t)$ (in blue), and the envelope $V_{\max }=f(t)$ (in red), and the corresponding friction law diagram $\mu=f\left(V_{\max }\right)$, for two normal loads $\mathrm{N}$ : (a) $25 \mathrm{mN}$, (b) $300 \mathrm{mN}$, and (c) $500 \mathrm{mN}$, for thin ILPB grafted on steel, that was tested by a steel sphere, at $25^{\circ} \mathrm{C}$.

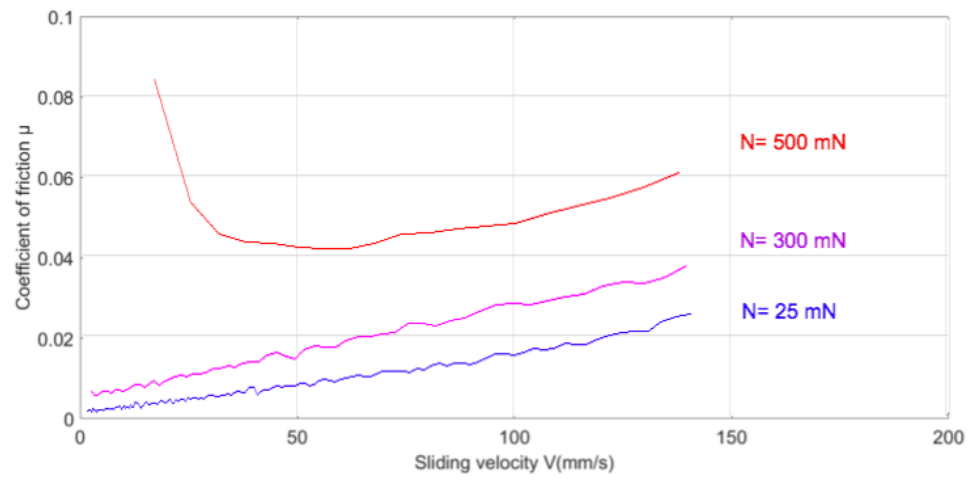

Figure 6. The Friction laws $\mu=f\left(V_{\max }\right)$ that were obtained on the thin ILBP that was grafted onto steel coupons. Friction was low and essentially viscous-type for the tests at normal force $N=25 \mathrm{mN}$ (blue) and $300 \mathrm{mN}$ (pink). In the case of $500 \mathrm{mN}$ applied load (red), we found a combination of viscous and Coulomb type contributions to friction, and a change in lubrication regime. 


\subsection{A New Lubrication Process}

The boundary lubrication regime of a lubricated tribosystem was generally described by the so-called 'Stribeck curve', giving a qualitative description of friction with a parameter that was strongly dependent on the sliding velocity. We found that the ILPBs that were grafted onto steel exhibited such a behavior, see Figure 6, with the friction having increased significantly when the sliding velocity decreased, to below $50 \mathrm{~mm} / \mathrm{s}$, in the example shown in Figure 6, for the results that were obtained for a normal load of $500 \mathrm{mN}$.

But the main result stemmed from the ILPBs that were grafted onto silicon. In this case, we showed that a Newtonian behavior was maintained, even when the sliding velocity reached a null value (the minimum here is $1.0 \mathrm{~mm} / \mathrm{s}$ ). Therefore, we showed that ILPBs were able to maintain a viscous-type interface, even when the sliding velocity reached zero. This point represented a new lubrication process, which led to a smooth viscous state, even at a high contact pressure.

\subsection{Durability of the ILPB Coating}

In order to check the resistance of this soft coating that was grafted onto a surface, the different experiments were conducted with multi-shoot relaxation tests on the same friction track, without unmounting the samples or changing any parameter in the contact. Here, we presented the results that were obtained on a thick ILPB that was grafted onto silicon coupons. The normal load was fixed at $500 \mathrm{mN}$, which corresponded to a maximum Hertzian pressure of $540 \mathrm{MPa}$.

Figure 7 shows the evolution of the number of oscillations that were observed in the different shoots (from 1 to 90). This number was found to be very stable (57.5 \pm 0.5 ), which showed that the damping at the interface remained strictly constant over the whole experiment (i.e., more than 5000 cumulated oscillations). This result was in good agreement with previous ones, which were already reported by Arafune et al. [12]. It confirmed the considerable potential of this technique for achieving low friction and good resistance for soft coatings.

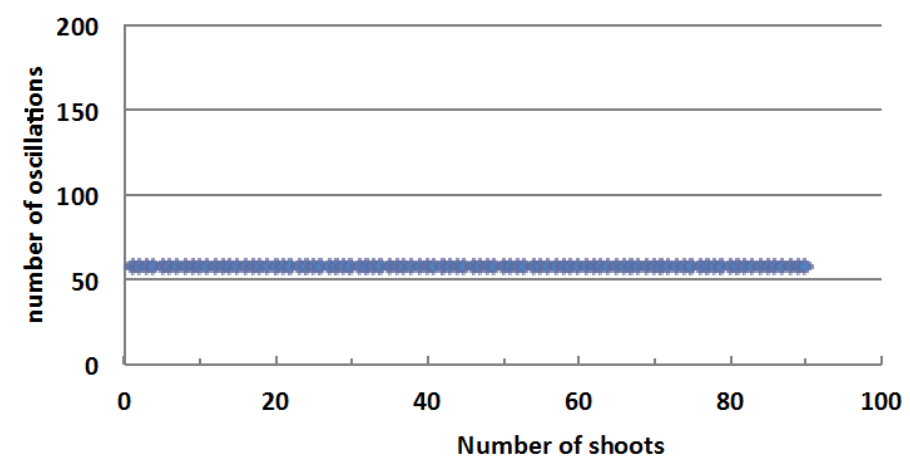

Figure 7. Evolution of the number of oscillations in a multi-shoot relaxation experiment: thick ILPB grafted onto silicon, tested by a steel sphere, normal load $500 \mathrm{mN}, 25^{\circ} \mathrm{C}$.

\section{Conclusions}

We presented original results showing the remarkable capacity of the ionic liquid-type polymer brushes (ILPBs) to achieve low friction. These coatings were elaborated on both silicon and steel substrates and were tested using the innovative relaxation tribometer technique, in which the precision on the friction value quantification increased as the friction decreased. We showed that the following was true:

- a thick layer of the PBs layer on the silicon was very resistant to high contact pressure, up to $540 \mathrm{MPa}$;

- the friction behavior of the brushes was close to a Newtonian viscous behavior, even at the maximum normal loads; 
- $\quad$ the corresponding friction law was viscous-type, even at a very low sliding velocity;

- $\quad$ poorer results were obtained on the thinner sample. The corresponding friction laws of such a tribosystem were shown to be viscous-type at low contact pressure, after which a Stribeck-like behavior was exhibited at a higher pressure;

- $\quad$ repetition, of up to 5000 oscillations, on the same surface did not affect the friction damping of the contact, which showed the very good resistance of this film to friction under severe contact conditions;

- $\quad$ the feasibility of grafting onto steel surfaces was demonstrated. Thus, these ILPBs' coatings could act as a very good lubricant, providing a friction law that differs considerably from a classic Stribeck curve. The friction at near-zero velocity was found to be in the millirange and was even lower. In the case of low contact pressure testing, $\mu$ as low as 0.0008 could be determined. These ILPBs were found to be robust, even under repetitive testing;

- finally, based on our results presented in this paper, the thicker polymer brushes were found to be more resilient under severe conditions. However, the thicker samples needed more time and more reagent for synthesis, which led to higher cost. We are now trying to ascertain the optimal conditions by cooperating with several companies, so as to utilize the polymer brushes for industrial usage.

Author Contributions: For this research work, the contributions of the different authors are the following: Conceptualization, M.B., H.A., T.K. and T.S.; Methodology, M.B. and H.A.; Tribotesting, M.B. and H.A.; Data Analysis Technique, J.P.-L. and M.B.; Resources, T.M. and S.H.; Supervision, M.B. and T.S.; Draft Writing and Review, M.B., H.A. and T.S.

Funding: This research was partly funded by the Japan Science and Technology ACCEL program, Grant-in-Aid for Scientific Research (No. 15H03871, No. 17H04902), sponsored by the Ministry of Education, Culture, Sports, Science and Technology (MEXT) in Japan, and the CNRS, France for supporting the exchanges that made this work possible.

Acknowledgments: The authors would like to thank Nathan Gerbi, a student at LTDS, for his precious help in relaxation data processing.

Conflicts of Interest: The authors declare no conflict of interest.

\section{References}

1. Tannous, J.; Dassenoy, F.; Lahouij, I.; Le Mogne, T.; Vacher, B.; Bruhács, A.; Tremel, W. Understanding the Tribochemical Mechanisms of IF-MoS 2 Nanoparticles Under Boundary Lubrication. Tribol. Lett. 2011, 41, 55-64. [CrossRef]

2. Martin, J.M. Superlubricity of Molybdenum Disulfide. In Superlubricity; Springer: Berlin, Germany, 2007; pp. 207-225, ISBN 13-978-0-444-52772-1.

3. Heeb, R.; Bielecki, R.M.; Lee, S.; Spencer, N.D. Room-Temperature, Aqueous-Phase Fabrication of Poly(methacrylic acid) Brushes by UV-LED-Induced, Controlled Radical Polymerization with High Selectivity for Surface-Bound Species. Macromolecules 2009, 42, 9124-9132. [CrossRef]

4. Chen, M.; Briscoe, W.H.; Armes, S.P.; Klein, J. Lubrication at Physiological Pressures by Polyzwitterionic Brushes. Science 2009, 323, 1698-1701. [CrossRef] [PubMed]

5. Nomura, A.; Goto, A.; Ohno, K.; Kayahara, E.; Yamago, S.; Tsujii, Y. Controlled synthesis of hydrophilic concentrated polymer brushes and their friction/lubrication properties in aqueous solutions. J. Polym. Sci. Part A Polym. Chem. 2011, 49, 5284-5292. [CrossRef]

6. Tsujii, Y.; Nomura, A.; Okayasu, K.; Gao, W.; Ohno, K.; Fukuda, T. AFM studies on microtribology of concentrated polymer brushes in solvents. J. Phys. Conf. Ser. 2009, 184, 12031. [CrossRef]

7. Nomura, A.; Ohno, K.; Fukuda, T.; Sato, T.; Tsujii, Y. Lubrication mechanism of concentrated polymer brushes in solvents: Effect of solvent viscosity. Polym. Chem. 2012, 3, 148. [CrossRef]

8. Kaneko, D.; Tada, T.; Kurokawa, T.; Gong, J.P.; Osada, Y. Mechanically Strong Hydrogels with Ultra-Low Frictional Coefficients. Adv. Mater. 2005, 17, 535-538. [CrossRef]

9. Torimoto, T.; Tsuda, T.; Okazaki, K.I.; Kuwabata, S. New frontiers in materials science opened by ionic liquids. Adv. Mater. 2010, 22, 1196-1221. [CrossRef] [PubMed] 
10. Minami, I. Ionic liquids in tribology. Molecules 2009, 14, 2286-2305. [CrossRef] [PubMed]

11. Ishikawa, T.; Kobayashi, M.; Takahara, A. Macroscopic frictional properties of poly(1-(2-methacryloyloxy) ethyl-3-butyl imidazolium bis(trifluoromethanesulfonyl)-imide) brush surfaces in an ionic liquid. ACS Appl. Mater. Interfaces 2010, 2, 1120-1128. [CrossRef] [PubMed]

12. Arafune, H.; Kamijo, T.; Morinaga, T.; Honma, S.; Sato, T.; Tsujii, Y. A Robust Lubrication System Using an Ionic Liquid Polymer Brush. Adv. Mater. Interfaces 2015, 2, 1500187. [CrossRef]

13. Drobek, T.; Spencer, N.D. Nanotribology of Surface-Grafted PEG Layers in an Aqueous Environment. Langmuir 2008, 24, 1484-1488. [CrossRef] [PubMed]

14. Brady, M.A.; Limpoco, F.T.; Perry, S.S. Solvent-dependent friction force response of poly(ethylenimine) -graft-poly(ethylene glycol) brushes investigated by atomic force microscopy. Langmuir 2009, 25, 7443-7449. [CrossRef] [PubMed]

15. Kitano, K.; Inoue, Y.; Matsuno, R.; Takai, M.; Ishihara, K. Nanoscale evaluation of lubricity on well-defined polymer brush surfaces using QCM-D and AFM. Colloids Surf. B Biointerfaces 2009, 74, 350-357. [CrossRef] [PubMed]

16. Ramakrishna, S.N.; Cirelli, M.; Divandari, M.; Benetti, E.M. Effects of Lateral Deformation by Thermoresponsive Polymer Brushes on the Measured Friction Forces. Langmuir 2017, 33, 4164-4171. [CrossRef] [PubMed]

17. Banquy, X.; Burdyńska, J.; Lee, D.W.; Matyjaszewski, K.; Israelachvili, J. Bioinspired bottle-brush polymer exhibits low friction and amontons-like behavior. J. Am. Chem. Soc. 2014, 136, 6199-6202. [CrossRef] [PubMed]

18. Zoppe, J.O.; Ataman, N.C.; Mocny, P.; Wang, J.; Moraes, J.; Klok, H.A. Surface-Initiated Controlled Radical Polymerization: State-of-the-Art, Opportunities, and Challenges in Surface and Interface Engineering with Polymer Brushes. Chem. Rev. 2017, 117, 1105-1318. [CrossRef] [PubMed]

19. Majdoub, F.; Belin, M.; Martin, J.M.; Perret-Liaudet, J.; Kano, M.; Yoshida, K. Exploring low friction of lubricated DLC coatings in no-wear conditions with a new relaxation tribometer. Tribol. Int. 2013, 65, 278-285. [CrossRef]

20. Majdoub, F.; Perret-Liaudet, J.; Belin, M.; Martin, J.M. Decaying law for the free oscillating response with a pseudo-polynomial friction law: Analysis of a superlow lubricated friction test. J. Sound Vib. 2015, 348, 263-281. [CrossRef]

21. Belin, M.; Kakizawa, M.; Martin, J.M. Dual characterization of boundary friction thanks to the harmonic tribometer: Identification of viscous and solid friction contributions. J. Phys. Conf. Ser. 2010, 258, 012008. [CrossRef]

22. Hriouech, A.; Belin, M.; Perret-Liaudet, J.; De Barros, M.I.; Diaby, M. An original tribometer coupling a free-oscillation dynamic tribotest with a conventional linear reciprocating tribometer. In Proceedings of the World Tribology Conference 2017, WTC'2017, Beijing, China, 18-22 September 2017. Poster ST-A-019. 